\title{
Études entomologique et typologique des gîtes larvaires des moustiques (Diptera : Culicidae) dans la région de Bousaâda (Algérie)
}

\section{Entomological and typological studies of larval breeding sites of mosquitoes (Diptera: Culicidae) in Bousaâda area (Algeria)}

Manuscrit reçu le 16 février 2018 et accepté le 14 juin 2018

$$
\text { BENHISSEN Saliha }{ }^{1 *} \text {, HABBACHI Wafa }{ }^{2} \text {, ReBBAs Khellaf }{ }^{13} \text {, MASNA Fatiha }{ }^{4}
$$

${ }^{1}$ Département des sciences de la nature et de la vie, faculté des sciences, université Mohamed Boudiaf de M'Sila, 28000, Algérie.

2 Laboratoire Neuroendocrinologie Appliquée. Département de Biologie, Faculté des Sciences, BP 12 Université Badji Mokhtar, 23000 Annaba, Algérie.

${ }^{3}$ Laboratoire d'Agro-Biotechnologie et de nutrition en zones arides et semi arides, Équipe de recherche de gestion des ressources naturelles et environnement. Université Ibn Khaldoun, Tiaret, Algérie.

${ }^{4}$ Laboratoire de Désertification et climat, Université Amar Telidji, 03000 Laghouat, Algérie

\section{Résumé}

Les moustiques ont un rôle épidémiologique en tant que vecteur de plusieurs agents pathogènes capables d'affecter l'homme. L'inventaire des Culicidés durant la période de janvier à mai 2017 nous a permis de connaître leurs répartitions spatio-temporelles dans plusieurs stations de la région de Bousaâda (M'Sila). Les échantillonnages larvaires de moustiques ont été effectuées par d'une louche, dans les milieux urbain et rurale dans quatre localités situées dans différentes altitudes.

Les résultats d'identification montrent l'existence de 11 espèces dans la région étudiée (Culiseta longiareolata, Culiseta subochrea, Culex pipiens, Culex deserticola, Culex theileri, Culex laticinctus, Culex brumpti, Culex perexiguus, Anopheles cinereus, Anopheles multicolor, Uranotaenia unguiculata). Cet inventaire est complété par une analyse physicochimique de l'eau pour étudier la corrélation entre la densité larvaire des moustiques et la qualité de gîte.

Mots clés : Culicidae, inventaire, systématique, analyse physico-chimique.

\section{Abstract:}

Mosquitoes have an epidemiological role as a vector of several parasites which can affect humans. The inventory of Culicidae during the period from January to May 2017 allowed us to know their spatio-temporal distributions in several stations of the region of Bousaâda (M'Sila). The larvae samplings of mosquitoes were made by a ladle, in the urban and the rural areas infour localities located in different altitudes.

\footnotetext{
*Email adress: s.benhissen@yahoo.com
} 
The results obtained from this study showed the existence of 11 species in the studied region (Culiseta longiareolata, Culiseta subochrea, Culex pipiens, Culex deserticola, Culex theileri, Culex laticinctus, Culex brumpti, Culex perexiguus, Anopheles cinereus, Anopheles multicolor, Uranotaenia unguiculata). This inventory is complemented by a physico-chemical analysis of water to study the correlation between the mosquito larval density and the breeding sites quality.

Keywords: Culicidae, inventory, systematic, physico-chemical analysis.

\section{Introduction}

Certains groupes d'insectes peuvent transmettre à l'homme plusieurs agents infectieux, dont un bon nombre peut se révéler pathogènes. Les Culicidés ont le premier rôle dans la transmission des maladies à transmission vectorielle comme (Dengue, Zika et Fièvre à Virus west Nile) qui représentent, aujourd'hui, les problèmes de santé les plus graves dans le monde (OMS, 1999), avec des retombées énormes sur l'économie de nombreux pays et principalement les pays tropicaux (Hassaine, 2002). En Algérie il y a quelques alertes a été lancée par les responsables de la santé comme le signalement en 2016 de la présence du moustique tigre dans plusieurs quartiers de la capitale, le comité national des arboviroses s'est mis en état d'alerte pour suivre l'évolution de la situation.

L'approche la plus efficace de démoustication demeure celle basée sur l'élimination ou la réduction des gîtes larvaires de moustiques, parce qu'à ce stade les moustiques occupent un espace géographique minimal et sont facilement localisables et contrôlables. Cette lutte doit être adaptée aux facteurs suivants : l'identification correcte de l'espèce, la connaissance de leurs gîtes larvaires, de leurs comportements et de leur écologie qu'est fondamentale pour assurer l'efficacité de cette action (Djogbénou, 2009).

Dans cette optique nous avons prospecte un inventaire des population culicidiennes dans les oasis de Bousaâda (M'sila, Algérie), par une étude systématique et biotypologie des gîtes larvaires. Les résultats de notre étude serviront pour la planification des futures compagnes de lutte contre les moustiques dans la région de Bousaâda.

\section{Matériels et méthodes}

\subsection{La zone d'étude}

La région de Bousaâda se situe au sud-est du nord algérien $\left(4^{\circ} 11^{\prime} \mathrm{N}, 35^{\circ} 13^{\prime} \mathrm{E}\right.$ et $560 \mathrm{~m}$ d'altitude) (Figure 1).

Elle se situe entre le littoral algérien et le désert et plus exactement entre la steppe du Hodna et les monts des Zibans. Elle est enserrée par deux montagnes dont la direction est nord-est sud-ouest. L'étage bioclimatique de la région est aride à hiver doux, avec une moyenne annuelle de précipitation de 178,9 $\mathrm{mm}$. La moyenne minimale de température annuelle du site est enregistrée en novembre avec $10,7^{\circ} \mathrm{C}$ et la moyenne maximale est celle du mois de juillet par $32,2^{\circ} \mathrm{C}$.

Les stations (S1, S2, S3 et S4) qui ont fait l'objet de l'étude ont été choisies de façon à avoir une représentativité, en tenant compte de leurs caractères écologiques à savoir milieu urbain, 
périurbain, rural, présence de l'eau permanente ou saisonnière, eau courante ou stagnante (Tableau 1).

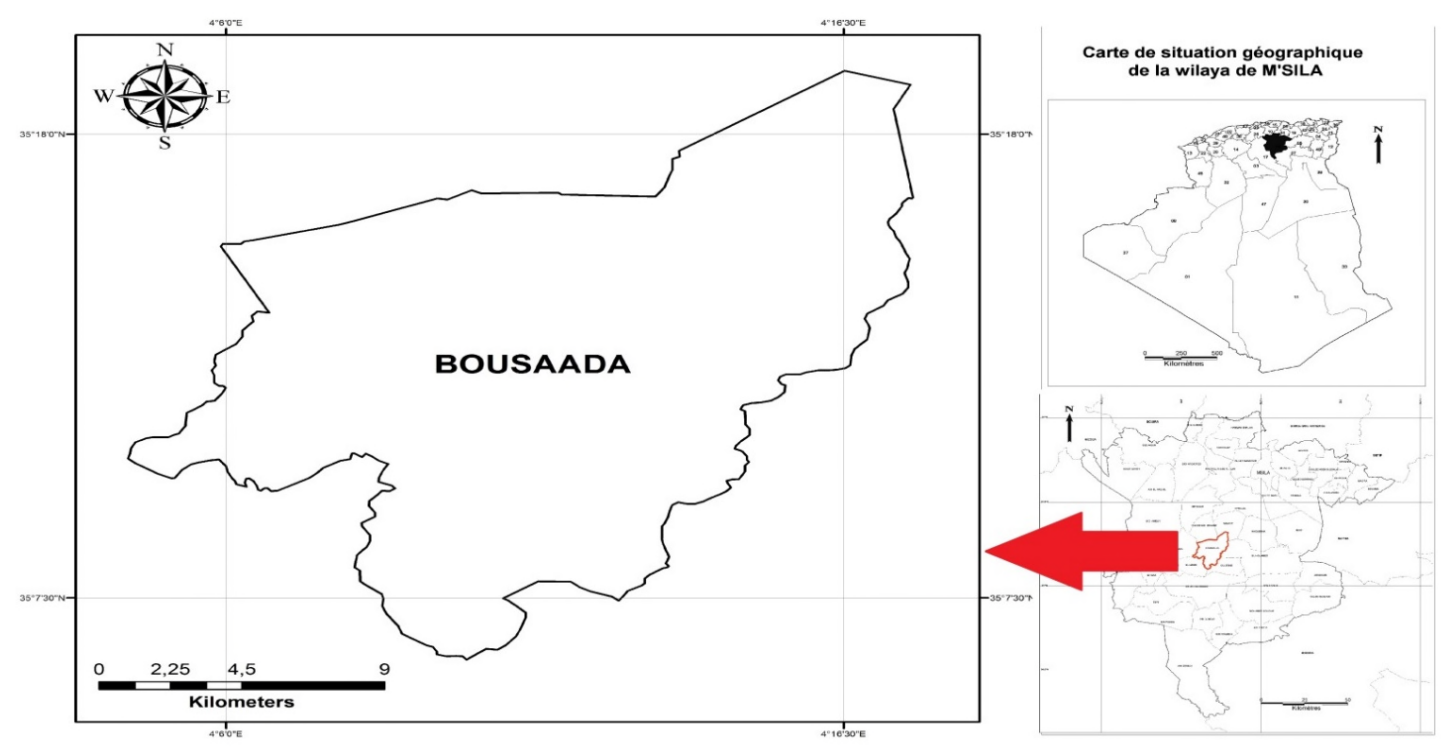

Fig.1 : Localisation géographique de la zone d'étude

Tableau 1 : Coordonnées géographiques des différentes stations inventoriées

\begin{tabular}{|c|c|c|c|c|}
\hline Site & Type & Latitude & Longitude & Altitude \\
\hline S1 & Oasien & $1^{\circ} 4^{\prime} 68.07^{\prime}$ & $12^{\circ} 35^{\prime} 24.49^{\prime}{ }^{\prime}$ & $573 \mathrm{~m}$ \\
\hline S2 & Urbaine & $1^{\circ} 4^{\prime} 36.38^{\prime}{ }^{\prime}$ & $13^{\circ} 35^{\prime} 16.16^{\prime}$ & $534 \mathrm{~m}$ \\
\hline S3 & Rural & $0^{\circ} 4^{\prime} 52.38^{\prime}{ }^{\prime}$ & $09^{\circ} 35^{\prime} 35.21^{\prime}{ }^{\prime}$ & $730 \mathrm{~m}$ \\
\hline S4 & Rural & $1^{\circ} 4^{\prime} 86.31^{\prime}{ }^{\prime}$ & $08^{\circ} 35^{\prime} 77.300^{\prime}$ & $701 \mathrm{~m}$ \\
\hline
\end{tabular}

\subsection{Sites prospectés :}

- (Oued Araga) (S01) : Site permanant, c'est une mare qui est alimentée par les crues de Oued Araga, riche en végétation avec des algues près des palmeraies (Fig. 1).

- (Bassin) (S02): Site urbaine, temporaire, c'est un bassin ouvert au sein d'une maison qui sert au stockage de l'eau d'irrigations. Ce basin est d'une superficie de $3 \mathrm{~m}^{2}$ et d'une profondeur de 1, $5 \mathrm{~m}$ (Fig. 2).

- Oued Magtaa (S3) : Site permanant éloignée 11Km à Bousaâda, c'est une mare dans l'Oued Magtaa, l'eau de ce site est composée par l'eau usée et de débordement de l'oued. Le couvert végétal est caractérisé par des arbres, les algues et des palmiers dattier (Fig. 3).

- Oued Sehil (S4) : Site temporaire éloignée 15Km à Bousaâda, c'est une mare dans l'Oued Sehil, qui est alimentée par les crues d'Oued Sehil. Le couvert végétal est caractérisé par des herbes Defla et les roseaux, des palmiers dattier (Fig. 4). 


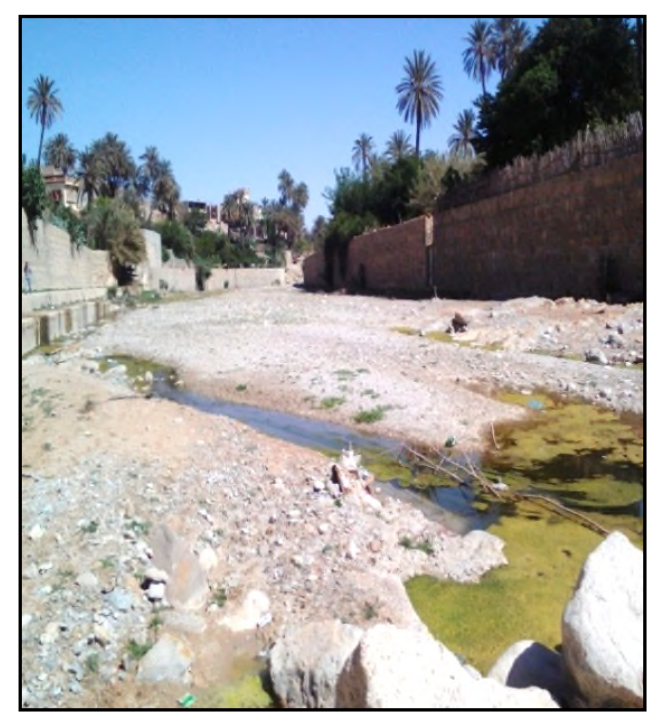

Figure 1 : Oued Araga (S1)

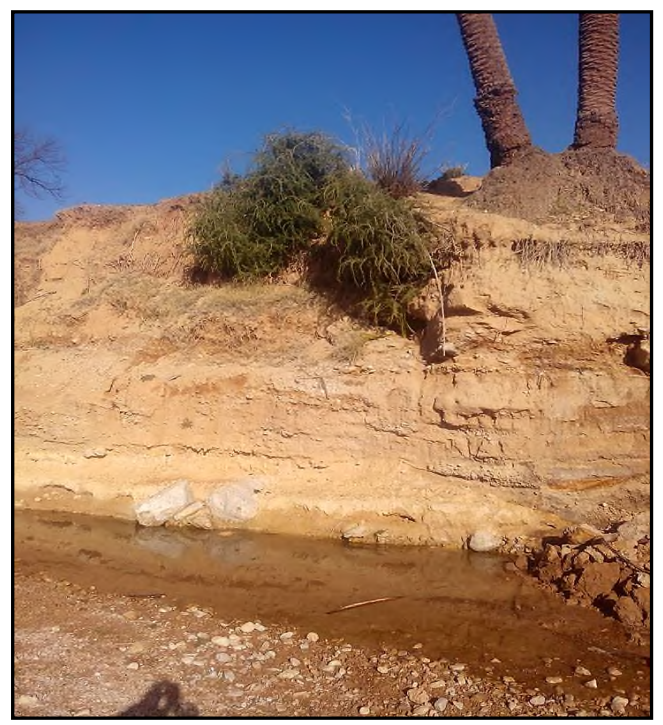

Figure 4 : Oued Magtaa (S3)

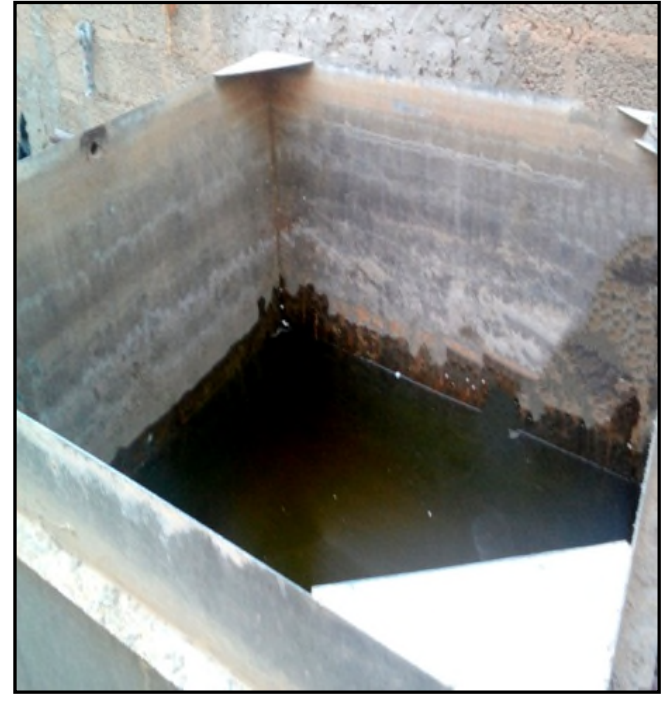

Figure 2 : Bassin (S2)

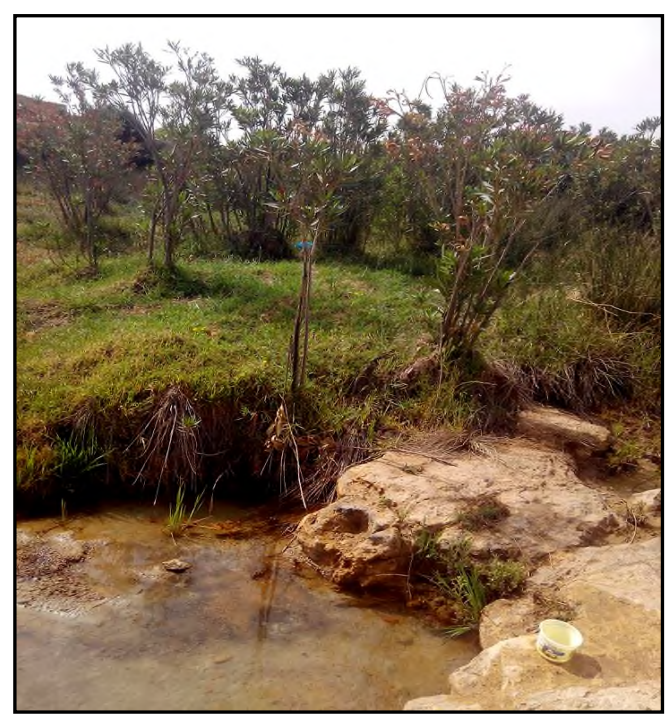

Figure 5 : Oued Sehil (S4)

Les larves de moustiques sont récoltées au niveau des plusieurs gîtes à l'aide d'une louche, que nous l'avons plongé un certain nombre de fois en des points déterminés du gîte et que nous avons soigneusement reparti dans deux récipients numérotés. Des larves de moustiques ramenées au laboratoire, une partie d'entre elles est mortes et elles seront conservées dans la glycérine puis identifiées et les vivantes vont servir à un élevage de masse au laboratoire pour obtenir les stades adultes.

La détermination des espèces culicidiennes a été faite à l'aide d'une loupe binoculaire, un microscope optique et le logiciel d'identification des moustiques de l'Afrique méditerranéenne (Brhunes et al., 1999) qui permet l'identification en se basant sur un ensemble de critères et de descripteurs microscopiques très précis pour les larves identifiées. 
Quatre paramètres physico-chimiques ( $\mathrm{pH}$, conductivité, température, TDS) sont mesurés au laboratoire après prélèvement d'eau dans chaque site. Les différents paramètres de l'analyse physico-chimique de l'eau ont été mesurés à l'aide de l'appareil multi-paramètre HI 9812-5. L'impact du milieu sur la répartition des moustiques a été évalué par une GLM (modèle linéaire généralisé) sur le logiciel $\mathrm{R}$ version 3.4.3.

\section{Résultats et discussion}

\subsection{Distribution des moustiques par gîte larvaire et par espèce}

Le tableau 2 présente la répartition des larves de moustiques récoltées par des sites larvaires. Nous constatons que les moustiques existent dans tous les sites larvaires explorés. Le deux site 01 et site 03 sont les plus riches par des larves avec un taux $33.13 \%$ et 33,00\% respectivement (Tableau 2). Le site 1 est un vaste ensemble constitue des sous-gîtes larvaires avec des caractéristiques plus ou moins variées. Ce qui explique la présence quantitative et qualitative de toutes les espèces de moustique dans ce gîte. Autour de ce gîte, que ce soit en amont ou en aval, se sont créées des activités humaines permanentes. Culiseta longiareolata est l'espèce dominante en termes de quantité car elle représente plus de la moitié des moustiques récoltées. La forte dominance de cette espèce est liée au type du milieu. Culiseta longiareolata est capable de se développer dans des gîtes différents. Cette espèce présente une grande aptitude à coloniser des biotopes naturels ainsi que les gîtes artificiels, différents par leurs caractéristiques physiques (Hassaine, 2002 ; Messai et al, 2010).

Les autres sites sont présentés par un faible taux des larves. Ce ci explique par la nature de faune du ces derniers qui est marquée par la présence des insectes et des petits vers. La composition faunique influencerait le développement des larves de moustiques dans ce gîte (Saotoing et al., 2014).

Tableau 2 : Distribution des moustiques par site larvaire et par espèce

\begin{tabular}{|c|c|c|c|c|c|c|c|c|c|c|c|c|c|}
\hline$\sum_{i=n}^{E}$ & $\begin{array}{l}0 \\
0 \\
0 \\
0 \\
0 \\
\vdots \\
0 \\
0 \\
\vdots \\
0 \\
0 \\
0\end{array}$ & 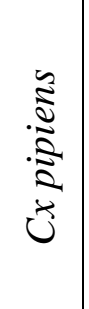 & 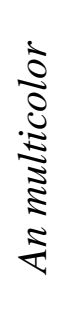 & 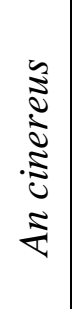 & 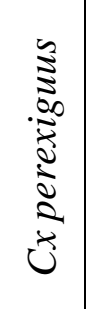 & 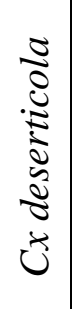 & 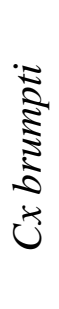 & 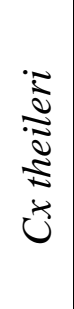 & 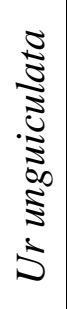 & 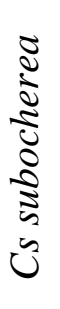 & 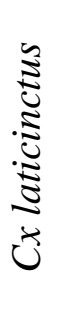 & 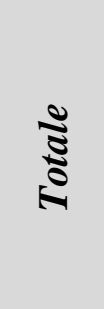 & $a^{2}$ \\
\hline S1 & 2203 & 428 & 6 & 10 & 104 & 6 & 6 & 27 & 11 & 5 & 2 & 2808 & 33.13 \\
\hline S2 & 1287 & 79 & 0 & 0 & 0 & 0 & 0 & 0 & 0 & 0 & 0 & 1366 & 16.12 \\
\hline S3 & 2791 & 0 & 0 & 0 & 0 & 0 & 0 & 6 & 0 & 0 & 0 & 2797 & 33.00 \\
\hline S4 & 1505 & 0 & 0 & 0 & 0 & 0 & 0 & 0 & 0 & 0 & 0 & 1505 & 17.76 \\
\hline \multicolumn{12}{|c|}{ Totaux : } & 8476 & 100 \\
\hline
\end{tabular}

\subsection{Les paramètres physico-chimiques des eaux}

Les paramètres physico-chimiques des gîtes larvaires étudiés montrent des grandes variations spatio-temporelles. Les valeurs moyennes sont rassemblées dans le Tableau 3. Les mesures de 
PH d'eau dans les différents sites de récolte varient entre 8 et 10 ce qui indique que l'eau de la région est alcaline. Le $\mathrm{pH}$ de l'eau reflète également l'activité biologique du milieu. Une alcalinisation du milieu peut être causée par une hausse de l'activité photosynthétique dans le lac (Serghini et $a l ., 2010$ ).

Par ailleurs le total de matière solide (TDS) des eaux analysées est, d'une façon générale, très fort dans les sites échantillonnés (varie de 1366 à 2808 ppm) (Tableau 3). La conductivité d'eau dans les sites est d'une moyenne de $1,19 \pm 0,39 \mu \mathrm{S} / \mathrm{cm}$ à un minimum de $1 \mu \mathrm{S} / \mathrm{cm}$ dans les quatre sites. En ce qui concerne la température, elle varie de 21 à $24{ }^{\circ} \mathrm{C}$, favorable au développement de plusieurs espèces de moustiques.

Le rôle de caractères physico-chimiques de l'eau est évident sur la répartition des espèces culicidienne. Ainsi, les espèces choisiront entre des milieux halophiles ou d'eau douce comme l'An multicolor. Précisément, il a été démontré que l'induction de l'éclosion pour certaines espèces était conditionnée par la qualité physicochimique de l'eau du gîte (Sinegre, 1974). Bien que ce facteur soit important, il est délicat d'en décrire le rôle exact. La conductivité, le $\mathrm{pH}$, la salinité, l'oxygène et le taux de bactérie sont apparus comme des paramètres importants, pouvant expliquer l'absence des larves dans certaines gîtes (Sérandour, 2009).

Tableau 3 : Paramètres physicochimiques relevées dans les sites d'étude.

\begin{tabular}{|c|c|c|c|c|c|}
\hline Les sites & $\mathbf{p H}$ & Conductivité & Température & Tds & Nombre des larves \\
\hline Site $\mathbf{1}$ & 8,3 & 1 & 24 & 1110 & 2808 \\
\hline Site 2 & 8,3 & 1,78 & 23 & 850 & 1366 \\
\hline Site 3 & 10,4 & 1 & 24 & 1030 & 2797 \\
\hline site 4 & 6,7 & 1 & 21 & 1750 & 1505 \\
\hline Moy $\mathbf{~ s ~}$ & $8,42 \pm 1,52$ & $1,19 \pm 0,39$ & $23 \pm 1,41$ & $1185 \pm 392$ & 8476 \\
\hline
\end{tabular}

S1 : Araga; S2 : Bassin; S3: Magtaa; S4 : Sehil ; Moy \pm s : moyenne \pm écart-type

Les composantes physico-chimiques d'une eau peuvent jouer un rôle primordial, non seulement dans la biologie d'une espèce mais aussi dans la structure et la dynamique de la biocénose toute entière (Berchi, 2000). D'après la figure 2, nous remarquons corrélation positive très faible entre le nombre des larves et $\mathrm{Tds}$ des eaux des sites $(\mathrm{R}=0.27)$. Les résultats montrent, aussi, qu'il y a une corrélation positive entre le nombre des individus récoltés (larves) et les autres paramètres étudiés $(\mathrm{pH}$, conductivité et température) $(\mathrm{R}=0.63$ à $0.77)$.

Le développement des Culicidaes est conditionné par la température et par la composition biologique ou chimique de l'eau (Messai et al., 2011). Les résultats obtenus dévoilent l'importance des deux paramètres la température et le PH sur l'apparition de la fluctuation des populations larvaires des moustiques (Fig. 6). De même, la température reste primordiale quant à l'accentuation de la vitesse de développement du moustique (Hadj et al., 2013).

Les paramètres mesurés dans l'eau des divers sites étudiés n'ont pas montré de différences significatives, ce qui peut expliquer la répartition inéquitable des espèces entre les sites. Ces facteurs, tels que le $\mathrm{pH}$ et la présence de sels dissous, sont moins fortement corrélés à la densité larvaire et ne sont probablement pas des facteurs déterminants (Kamura, 1959). 


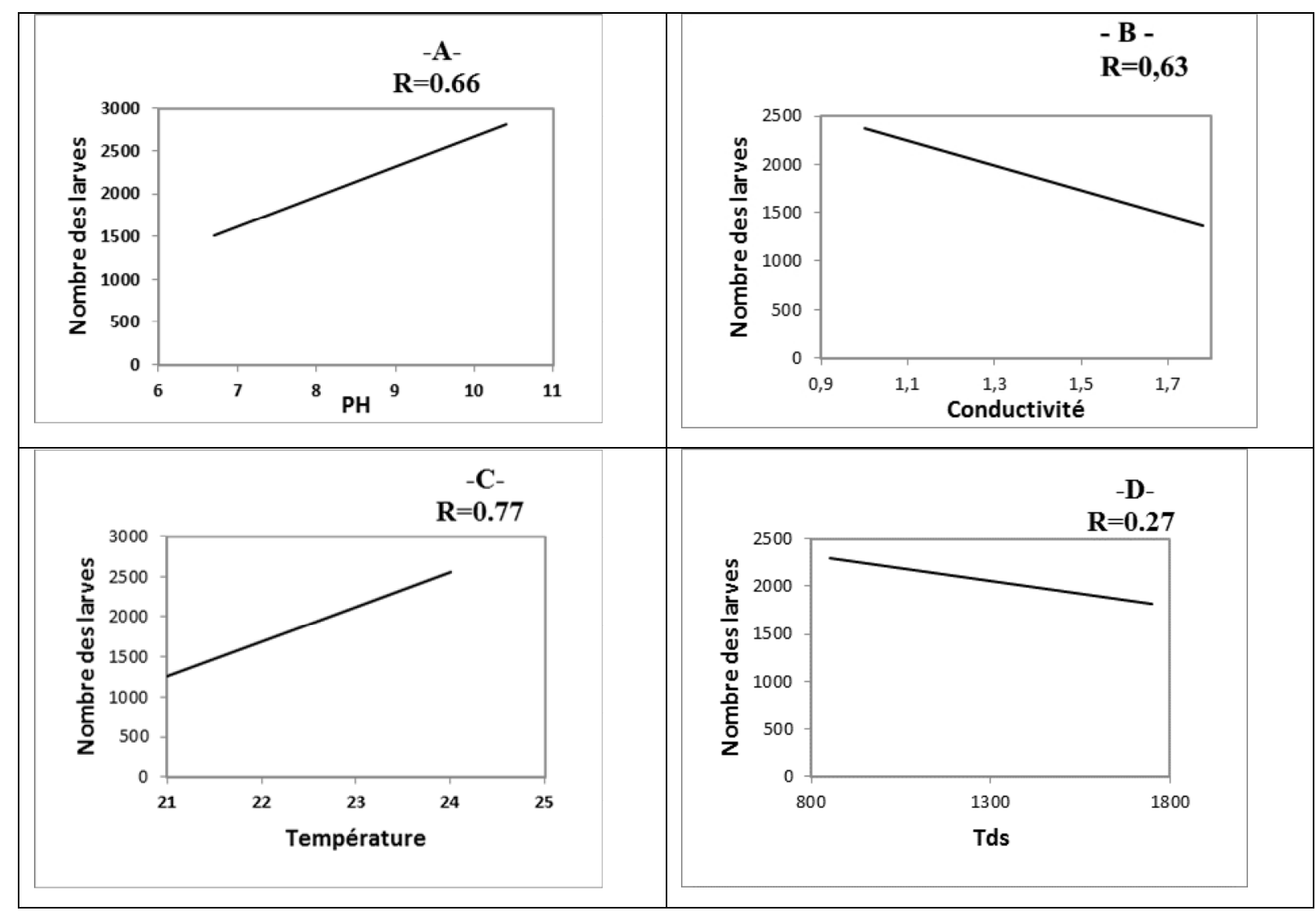

Fig. 6 : Relation entre nombre des larves et les paramètres physico-chimiques des eaux.

Les résultats montrent un effet significatif entre la nature de gîte et sur l'abondante et la répartition des espèces ( $p: 0.034)$ (Tableau 4). Nos résultats concordent avec ceux de (Messai \& al., 2010) et (Brahmi \& al., 2007) qui ont jugé que le biotope primordial pour la prolifération de moustiques résulte de l'interaction entre plusieurs composantes environnementales comme l'eau, les sols hydro morphes et salés et la végétation qui leur est associée.

La répartition de l'espèce, la mieux représentée dans la région de Bousaâda, Cs. longiareolata est fortement liée à ce facteur (nature de gîte) ( $p: 0.015)$ (Tableau. 4). Culiseta longiareolata peuple les gîtes les plus divers, son optimum écologique est atteint dans les eaux claires (Hassaine, 2002 ; Himmi, 2007). En outre, cette espèce a été récoltée par Berner (1974) et Hassaine (2002) dans les eaux riches en matière organique. Ceci concorde avec les résultats obtenus dans notre région. 
Tableau 4 : La répartition du moustique en fonction des gîtes et sites d'étude

\begin{tabular}{|l|c|c|c|c|c|c|c|c|}
\hline \multicolumn{1}{|c|}{ Factors } & \multicolumn{4}{|c|}{ Habitat } & \multicolumn{4}{c|}{ Localities } \\
\hline & Estimate & Std. Error & $\mathbf{z ~ v a l u e}$ & $\operatorname{Pr}(>|\mathbf{z}|)$ & Estimate & Std. Error & $\mathbf{z}$ value & $\operatorname{Pr}(>|\mathbf{z}|)$ \\
\hline (Intercept) & 1.85 & 0.87 & 2.123 & $0.034 *$ & 0.56 & 0.97 & 0.577 & 0.564 \\
\hline An.cinereus & -123.88 & 25240.58 & -0.005 & 0.996 & 136.73 & 23064.68 & 0.006 & 0.995 \\
\hline An.multicolor & -53.62 & 10308.22 & -0.005 & 0.996 & 58.30 & 9469.86 & 0.006 & 0.995 \\
\hline Cs.longiareolata & -0.011 & 0.005 & -2.422 & $0.015 * *$ & 0.006 & 0.0058 & 1.127 & 0.260 \\
\hline Cs.subochrea & 35.059 & 12994.005 & 0.003 & 0.998 & -39.53 & 12558.98 & -0.003 & 0.997 \\
\hline Cx.brumpti & NA & NA & NA & NA & NA & NA & NA & NA \\
\hline Cx.déserticola & NA & NA & NA & NA & NA & NA & NA & NA \\
\hline Cx.laticinctus & NA & NA & NA & NA & NA & NA & NA & NA \\
\hline Cx.perexiguus & NA & NA & NA & NA & NA & NA & NA & NA \\
\hline Cx.pipiens & 3.297 & 566.14 & 0.006 & 0.995 & -3.61 & 504.10 & -0.007 & 0.994 \\
\hline Cx.theileri & -3.317 & 2955.06 & -0.001 & 0.999 & 3.094 & 2955.06 & 0.001 & 0.999 \\
\hline Ur.unguiculata & NA & NA & NA & NA & NA & NA & NA & NA \\
\hline
\end{tabular}

(NA : Not Attributed : le logiciel n'effectuent pas des analyses sur les données de ces espèces qui sont absentes dans la plupart des sites prospectés à l'exception du site 1 de Araga où sont considérées comme espèces accessoires ou rares)

\section{Conclusion}

Malgré l'aire limitée de notre prospection entomologique, il apparait d'après ce que nous avons obtenu, que la région de Bousaâda révèle une variété importante de moustique. Nous avons identifié 11 espèces de Culicidae, appartenant à deux sous familles : les Anophelinae et les Culicinae. Deux espèces d'Anopheles ont été identifiées: Anophèles multicolor, Anopheles cinereus et trois genres de la sous famille des Culicinae ont été identifiés: il s'agit de genre Culex, Culiseta et Uranotaenia.

Du point de vue densité, les espèces du genre Culiseta représentent une forte proportion des populations à Bousaâda. Ce genre de moustiques piquent de préférence les vertébrés surtout les oiseaux, très rarement l'humain, l'espèce est considérée comme un vecteur de Plasmodium d'oiseau.

\section{Reference bibliographiques}

BERNER L., 1974, Liste des moustiques provençaux. Bulletin du Muséum d'histoire.

BERCHI S., 2000, Bioécologie de Culex pipiens. (Diptera, Culicidae) dans la région de Constantine et perspective de lutte. Thèse Doc. Es-science. Université de Constantine. Algérie : 133p.

BRAHMI N., HATIRA A., RABIA M.C., TOUNSI I., BOUHAFA T et BACCAR L. 2007, Cartographie des zones à risque de prolifération des Aedes : application de l'approche géomatique dans la région de Bizerte (Tunisie). Revue Méditerranéenne de l'Environnement (2) pp 397-413.

BRUNHES I., RHAIM A., GEOFFROY B., ANGEL G. et HERVY J.P. 1999, Les moustiques de l'Afrique méditerranéenne. Logiciel d'identification et d'enseignement. I.R.D., édition. 2000. 
DJOGBENOU L., 2009, Lutte antivectorielle contre le paludisme et résistance des vecteurs aux insecticides en Afrique. Méd Trop 69(2):160-164

GUILLE G., 1976, Recherches éco-éthologiques sur Coquillettidia (Coquillettidia) richiardii (Ficalbi), 1989 (Diptera-Culicidae) du littoral méditerranéen français. II. Milieu et comportement. Annales de sciences naturelles, Zoologie et Biologie animale, 12e Série. 18 (1) : 5-112.

HADJI. M., BELGHYTI. D., EL ASSAL. M., ELOMARI. F. et RAHMOUN. H, 2013, Étude entomologique des gîtes larvaires des moustiques (Anophèles, Culex). Science lib. Édition Mersenne.

HASSAINE K., 2002, Biogéographie et biotypologie des Culicidae (Diptera - Nematocera) de l'Afrique méditerranéenne. Bioecologie des espèces les plus vulnérantes (Aedes caspius, Aedes destritus, Aedes mariae et Culex pipiens) de la région occidentale algérienne.Thèse Doc. d'État. Univ. Tlemcen: 203p.

HIMMI O., 2007, Les culicides (Insectes, Diptères) au Maroc : Systématique, écologique et études épidémiologiques pilotes. Thèse Doc d'État, Université Mohamed V, Faculté des Sciences de Rabat.

KAMURA T., 1959, Studies on the Culex pipiens group of Japan. IV. Ecological studies on the Nagasaki molestus. Endem. Dis. Bull. Nagasaki Univ., 1: 51-59.

MESSAI N., BERCHI S., BOULKNAFED F. et LOUADI K. 2010, Inventaire systématique et diversité biologique de Culicidae (Diptera: Nematocera) dans la région de Mila (Algérie). Entomologie faunistique .63(3), pp. 203-206.

OMS., 1999, La lutte anti vectorielle, méthode à usage individuel et communautaire. (449p). SAOTOING P., NJAN NLOGA A M., TCHUENGUEM FOHOUO F N., YAYA O. et MESSI J. 2014, Bio-ecologie des larves de Culicidae (Diptera) dans la ville de Maroua, Extreme-Nord du Cameroun. International Journal of Innovation and Applied Studies. , 39(1) : 438448.

SÉRANDOUR J., 2007, Contribution à l'étude des moustiques anthropophiles de France : le cas particulier du genre Coquillettidia.. Thèse Doc Université Joseph-Fourier - Grenoble I,. Français (213p).

SERGHINI A., FEKHAOUI M., EL ABIDI A., EL BLIDI S et BEN AKKAME R. 2010, Caractérisation hydrochimique d'un site Ramsar : le complexe zones humides de Mohammedia (Maroc). Bulletin de l'Institut Scientifique, Rabat, section Sciences de la Vie, 2010, n³2 (2), 133-145.

SINEGRE G., 1974, Contribution à l'étude physiologique d'Aedes (O) caspius (Pallas, 1771) (Nematocera-Culcidae). Éclosion, dormance, développement, fertilité. Thése és-Sciences, Uni. Sci.Tech. Languedoc, 285p. 